\title{
dCATCH-Seq: improved sequencing of large continuous genomic targets with double- hybridization
}

\author{
Yanfeng Zhang ${ }^{1}$, Jun Song ${ }^{1}$, Kenneth Day ${ }^{1}$ and Devin Absher ${ }^{1,2^{*}}$
}

\begin{abstract}
Background: Targeted sequencing is a powerful tool with broad application in both basic and translational sciences. Relatively low on-target rates for most current targeted sequencing studies influence the required coverage and data quality for subsequent applications.

Results: We present an improved targeted sequencing method that uses two rounds of in solution hybridization with probes synthesized from genomic clone templates, termed dCATCH-Seq. Independent captures of two large continuous genomic regions across three cell types within the human major histocompatibility complex (MHC) that spans $\sim 3.5 \mathrm{Mb}$ and a $\sim 250 \mathrm{~kb}$ region on chromosome 11 demonstrated that dCATCH-Seq was highly reproducible with $\sim 95 \%$ capture specificity. Comprehensive analyses of sequencing data generated using the dCATCH-Seq approach also showed high accuracy in the detection of genetic variants and HLA typing. The double hybridization capture approach can also be coupled with bisulfite sequencing for DNA methylation profiling of both $\mathrm{CpG}$ and non-CpG sites.
\end{abstract}

Conclusions: Altogether, dCATCH-Seq is a powerful and scalable targeted sequencing approach to investigate both genetic and epigenetic features.

Keywords: Targeted sequencing, Mhc, Hla, DNA methylation, Hybridization, Bac

\section{Background}

The primary design of targeted sequencing is to capture genetic variants within intended regions [1]. Some targets, such as exome capture, has been broadly used in both basic and translational research that includes characterization of genetic diversity and demographic history in human populations $[2,3]$, identification of etiological variants [4], crossspecies genome comparisons [5], and even phylogenetic estimation [6].

There are three main techniques [7] for targeted enrichment of DNA sequences that include hybridizationbased capture either in solution or on a solid support, e.g., TruSeq (Illumina), SureSelect (Agilent), and SeqCap (Roche NimbleGen) platforms [8-10], selective circularization, and PCR amplification. In principle, targeted sequencing approaches should efficiently capture DNA molecules within the intended genomic regions

* Correspondence: dabsher@hudsonalpha.org

${ }^{1}$ HudsonAlpha Institute for Biotechnology, Huntsville, USA

Ubiquity Genomics Inc., Huntsville, USA with little to no sequences outside these regions of interest. However, most of current targeted sequencing studies, including commercialized whole-exome sequencing, generally show a target specificity in a range from $40 \%$ to $80 \%$, and rarely approach $90 \%$, regardless of probes used in hybrid selection [9-15]. Although some off-target regions neighboring intended targets such as splice sites and intron edges in exome capture "splash", could be informative [16], the capture of the non-target regions at different levels may substantially impact data quality and adequate coverage across the intended targets, and eventually require more sequencing costs. Some strategies in measuring target efficiency have also been developed, e.g., SeqCap qPCR kit (Roche) and Multipoint Test for Targeted-enrichment Efficiency (MTTE) [17].

We previously reported the clone adapted template capture hybridization sequencing (CATCH-Seq) procedure that synthesizes probe sets from a pool of selected BAC or fosmid DNA templates for in solution hybrid capture [13]. We have employed an updated target capture strategy to improve on target capture efficiency of 
large genomic regions by two rounds of hybridization in solution that we term double CATCH-Seq (dCATCHSeq). To evaluate the performance of this procedure, we independently tested two large continuous genomic regions, including the entire major histocompatibility complex (MHC), covering a size of $3.5 \mathrm{Mb}$, using a custom probe set generated from a pool of 140 reference BAC DNAs. Our updated approach provides a more efficient alternative to the previously reported approach, and demonstrates the feasibility of capturing large and diverse genomic regions that enables new applications such as high resolution HLA typing.

\section{Results}

\section{Double versus single hybridization capture}

Synthesized BAC-based probes with an average size of $\sim 250$ bp were previously used for development of the CATCH-Seq procedure by in-solution hybridization [13, 14]. Following a protocol presented in the previous CATCH-seq, but undergoing two rounds of hybridization capture (see Methods), we referred to this strategy as dCATCH-Seq. We first compared the target efficiency of dCATCH-Seq versus a single round of capture using the standard CATCH-Seq method. One region was captured with a pool of probes using a BAC DNA as a template covering $\sim 248.6 \mathrm{~kb}$ on chromosome 11 (Fig. 1a). For both methods, we captured with at least five technical replicates along with $2 \times 100$ bp pairedend sequencing. A total of 170,700 and 140,200 reads on average were obtained across technical replicates for the single and double hybridizations, respectively, and on average $96.3 \%$ of the reads were mapped to the reference genome (Additional file 1: Table S1). The on target rate (target specificity) was calculated for all replicates by dividing the number of mapped reads within the target coordinates by the total number of mapped reads. On target rates were $92.2 \% \pm 2.65 \%$ and $37.2 \% \pm 4.34 \%$ (mean \pm s.d.) for the double and single hybridization approaches, respectively (Fig. 1b). We then merged the data across technical replicates for each approach, which resulted in over 100X coverage across the intended region (Table 1). The target specificity was more than doubled for dCATCH-Seq with each approach having identical sequencing depth. Using read depth covered within $200 \mathrm{bp}$ non-overlapping windows across the target site, dCATCH-Seq had an extended proportion of read bins covered with higher depth compared to single hybridization (Fig. 1c), regardless of the fraction of repeats (repeat-masked or not).

We next addressed whether two rounds of capture could introduce greater bias within the targeted intervals. We normalized read coverage between the two methods by calculating the read depth per 200 bp window divided by the total number of mapped reads within the entire target (with $\log _{2}$ transformation) to measure uniformity of coverage across the target in both approaches. Both approaches were highly concordant $\left(R^{2}=0.93, P<1 X 10^{-16}\right.$, Fig. $\left.1 d\right)$, which suggests no further regional bias was introduced from an additional capture in the dCATCH-Seq method.

We further conducted a comparative analysis between these two approaches in terms of the accuracy in the identification of genetic variants. In total, 259 single nucleotide variants (SNVs) were identified by two methods, where 253 were known single nucleotide polymorphisms (SNPs). Among them, 115 were homozygous SNVs. All were concordantly determined by both methods. Except for three discordantly identified SNVs located in the simple repeat (polyadenine) regions (Additional file 2: Figure S1), the remaining 144 heterozygous SNVs are concordantly called from the dCATCH-Seq data and the $\mathrm{CATCH}-\mathrm{Seq}$ data. Therefore, an estimation of the accuracy rate in variant calls for dCATCH-Seq was $~ 99.2 \%$ relative to the single hybridization approach. We also presented a comparison of the allelic depth for 141 heterozygous SNVs called from both methods. As Fig. 1e shows, the dCATCH-Seq approach did not introduce allelic bias relative to the single hybridization.

We also compared the Indel calls between the two methods. Of 32 Indels identified, $30(\sim 93.8 \%)$ showed a concordance of genotype calls (Additional file 1: Table S2). The remaining two discordantly called Indels were identified as heterozygous from the $\mathrm{dCATCH}-\mathrm{Seq}$ data, while as homozygous from the CATCH-Seq data (Additional file 2: Figure S2). Using independent $\mathrm{CATCH}$-seq assays in two replicates, we further validated that these two Indels are heterozygous insertions (Additional file 2: Figure S3).

\section{Double hybridization capture on the MHC region}

We next tested two conditions with or without PCR amplification after first round of capture using a synthesized probe set on a $\sim 3.5 \mathrm{Mb}$ continuous $\mathrm{MHC}$ region that covers the three major classes of MHC molecules with DNA libraries prepared from $\mathrm{K} 562$ cells (Fig. 2a). The sequencing data showed that both conditions were capable of capturing the entire $\mathrm{MHC}$ region with identically high target specificity (92.7\%) and alignment rate (97\%, Table 2). Comparative analyses based on both raw read coverage and normalized read coverage binned into $200 \mathrm{bp}$ windows also showed that the two conditions exhibited high concordance $\left(\mathrm{R}^{2}=0.972, P<1 \mathrm{X} 10^{-16}\right.$, Fig. $2 \mathrm{~b}$ and $\mathrm{c}$ ).

By comparing the efficiency of detecting structural variants (SVs) between the two conditions, we also found a similar performance for both (Additional file 2: Figure S4). In comparison with previously reported SVs from a whole-genome sequencing study in the same cell line [18], both methods could detect the majority of reported 


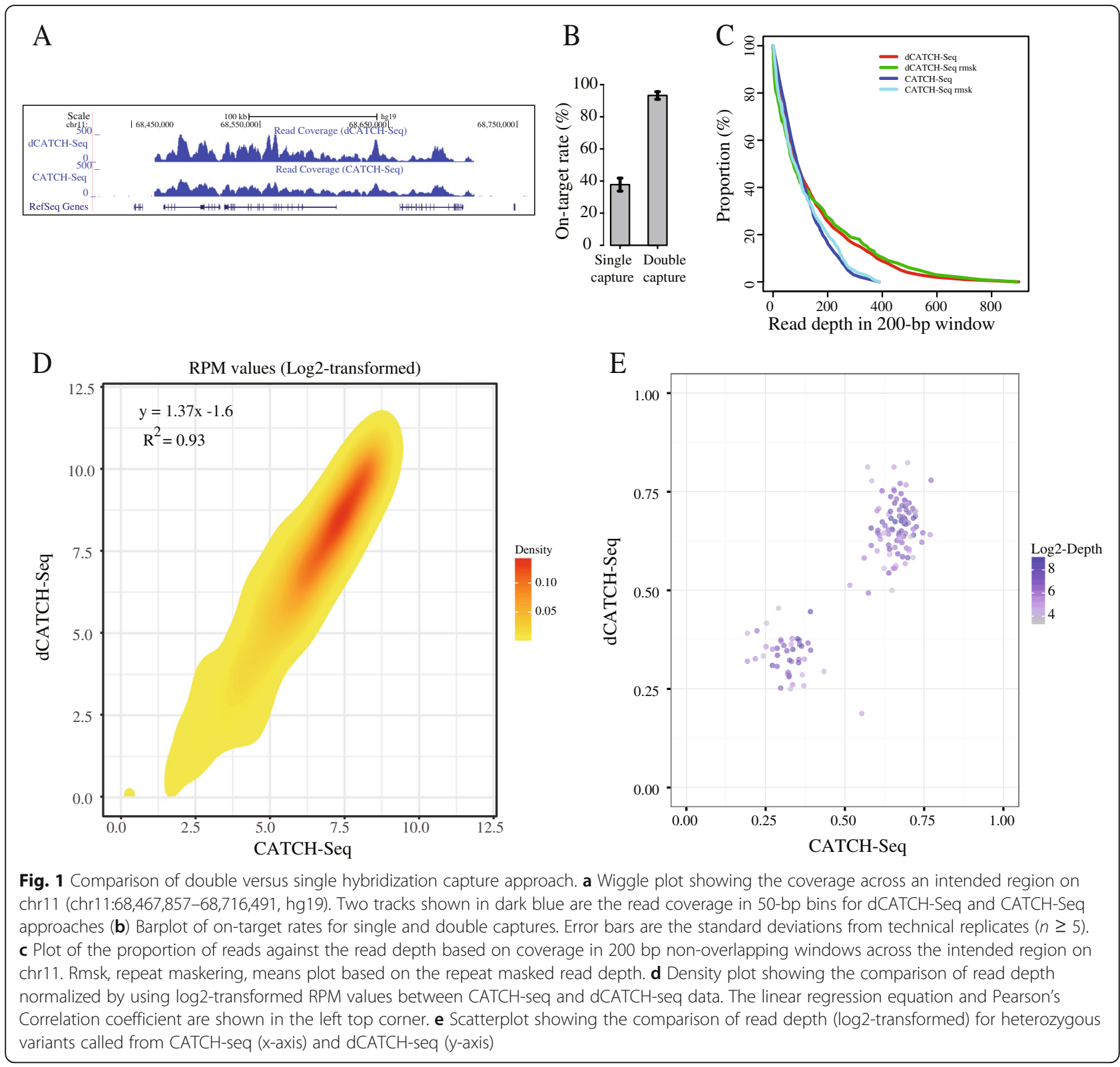

SVs across the MHC region (Additional file 2: Figure S4). In the present study, we also discovered two novel deletions in K562 cell genome. One was a homozygous deletion spanning $\sim 2.5 \mathrm{~kb}$ in size (Additional file 2: Figure S5A, B), and the other was an $\sim 800$ bp heterozygous deletion (Additional file 2: Figure S5C, D). Both deletions were confirmed by the PCR using primers that flanked the respective deletions (Additional file 2: Figure S5B and S5D). Altogether, our results suggest that PCR amplification of DNA library after the first hybridization capture is an optional step.

We again performed dCATCH-Seq on the human MHC region with no PCR amplification after the first hybridization capture with DNA libraries prepared from

Table 1 Summary of merged sequencing data in a comparison between dCATCH-Seq and CATCH-Seq

\begin{tabular}{|c|c|c|c|c|c|c|c|c|c|}
\hline Experiment & Total reads & Aligned reads & $\begin{array}{l}\text { Mean PCR } \\
\text { duplicates (\%) }\end{array}$ & $\begin{array}{l}\text { Aligned reads } \\
\text { on target }\end{array}$ & $\begin{array}{l}\text { On-target } \\
\text { rate }(\%)\end{array}$ & $\begin{array}{l}\text { Uniquely } \\
\text { aligned reads }^{a}\end{array}$ & $\begin{array}{l}\text { Uniquely aligned } \\
\text { reads on target }^{a}\end{array}$ & $\begin{array}{l}\text { On-target } \\
\text { rate }(\%)^{a}\end{array}$ & $\begin{array}{l}\text { Mean coverage } \\
\text { on target }\end{array}$ \\
\hline dCATCH-Seq & 853,358 & 414,965 & 47.4 & 382,625 & 92.21 & 406,331 & 379,084 & 93.29 & 145.98 \\
\hline $\mathrm{CATCH}-\mathrm{Seq}$ & 841,154 & 802,931 & 2.8 & 291,808 & 36.34 & 762,445 & 289,558 & 37.98 & 110.18 \\
\hline
\end{tabular}

${ }^{a}$ Uniqueness and relevant statistics are calculated from reads with mapping quality (MAPQ) $\geq 20$ 


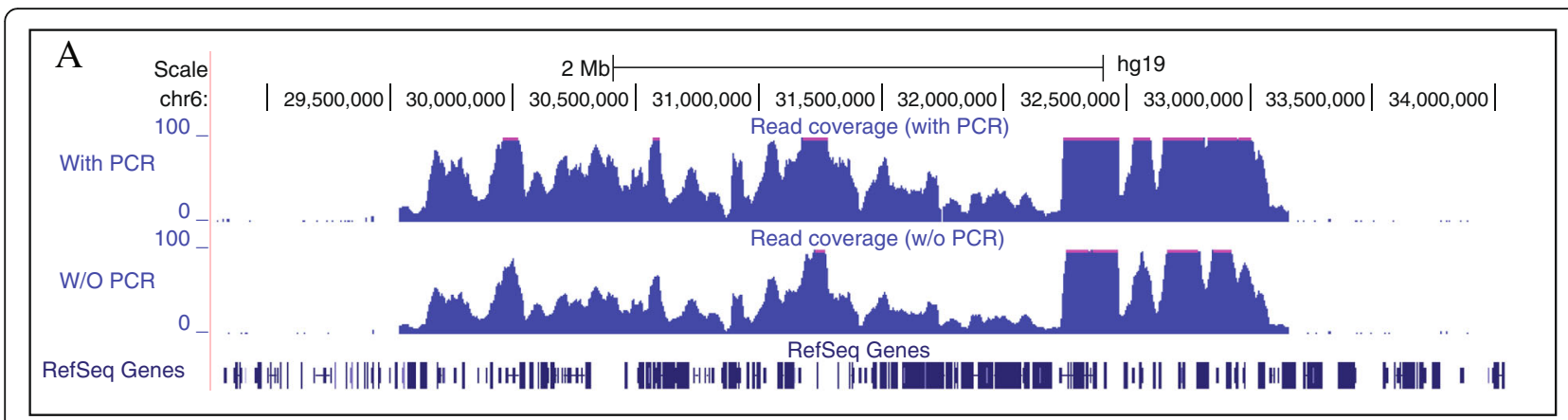

B

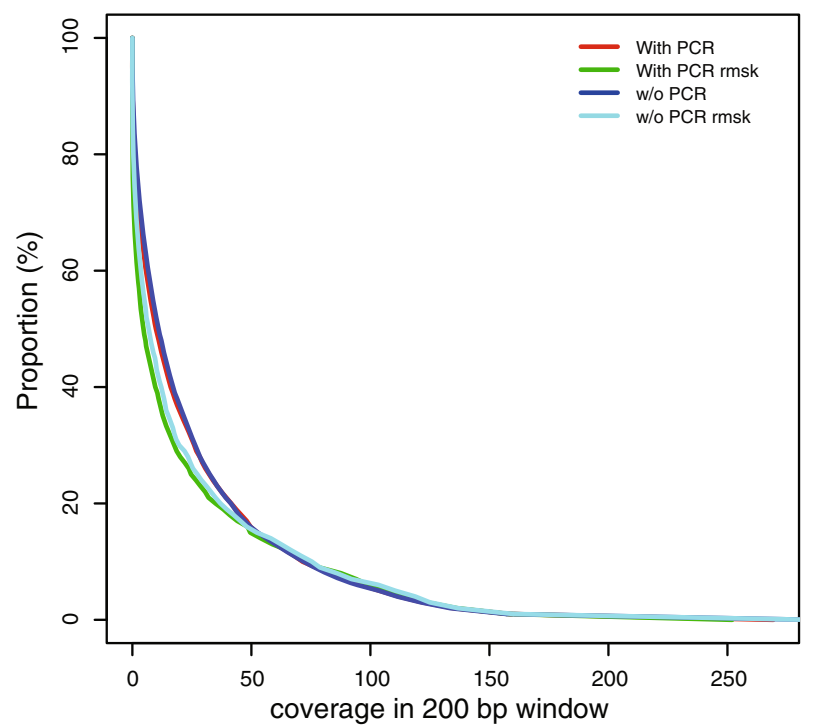

$\mathrm{C}$

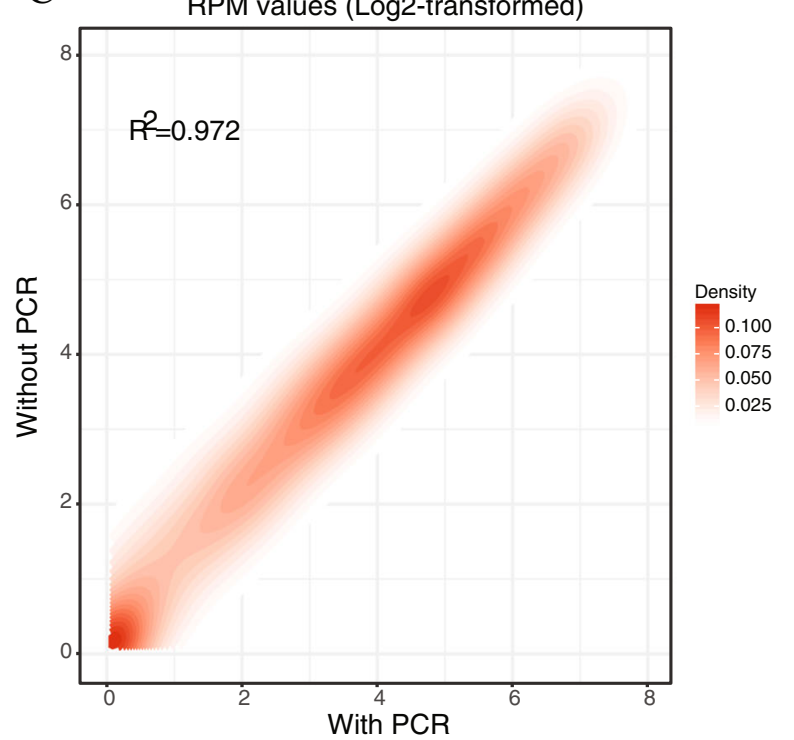

Fig. 2 Double capture and sequencing of the MHC region in K562 cells. a Wiggle plot showing the capture of the human MHC region with a size of $3.5 \mathrm{Mb}$ (chr6:29,651,300-33,160,000, hg19) under two conditions (with PCR amplification or not). b Plot of the proportion of reads against the read depth based on coverage in 200-bp non-overlapping windows across the MHC region for the two conditions. Rmsk: repeat masker. c Density plot showing the comparison of read depth normalized by using the log2-transformed RPM value between two conditions

GM12878 and U937 cells. Similar to results from K562 cells, we reproducibly captured the entire MHC region with a target specificity of $~ 90 \%$ (Fig. 3a, Additional file 2 : Figure S6 and Table 2) in these two cell genomes. A comparative analysis of the allelic depth for 8913 heterozygous SNVs identified across the MHC region for GM12878 cell lines showed that both alleles were almost equally captured (Fig. 3b). The result was consistent with a previous study showing that probes in long length (e.g.,
$>150$ bases) are tolerant to polymorphisms [19]. We also observed a similar result for the allelic depth of 7116 heterozygous SNVs called in U937 cells (Additional file 2: Figure S7). We further evaluated the accuracy of variant calls on the MHC region. Compared to microarray genotyping data, there were 277 SNPs within the sequenced target that overlapped with microarray probes. Among them, three SNPs (two were heterozygous and one homozygous in our dCATCH-Seq data) showed the discordance

Table 2 Summary of the MHC regional capture using dCATCH-Seq in three cell genomes

\begin{tabular}{|c|c|c|c|c|c|c|c|c|c|}
\hline Cell & Condition $^{\mathrm{a}}$ & Total reads & Aligned reads & $\begin{array}{l}\text { Alignment } \\
\text { rate (\%) }\end{array}$ & $\begin{array}{l}\text { PCR duplicate } \\
\text { rate (\%) }\end{array}$ & $\begin{array}{l}\text { Aligned reads } \\
\text { on target }\end{array}$ & $\begin{array}{l}\text { On-target } \\
\text { rate (\%) }\end{array}$ & $\begin{array}{l}\text { Uniquely aligned } \\
\text { reads on target }\end{array}$ & $\begin{array}{l}\text { On-target } \\
\text { rate }(\%)^{\mathrm{b}}\end{array}$ \\
\hline K562 & With PCR & $2,882,056$ & $2,744,145$ & 97.79 & 2.67 & $2,503,434$ & 91.23 & $2,342,653$ & 92.77 \\
\hline K562 & Without PCR & $1,922,388$ & $1,795,403$ & 97.67 & 4.43 & $1,633,616$ & 90.99 & $1,528,150$ & 92.71 \\
\hline GM12878 & Without PCR & $4,243,904$ & $3,836,649$ & 98.44 & 8.39 & $3,492,283$ & 91.02 & $3,228,811$ & 92.28 \\
\hline U937 & Without PCR & $1,489,332$ & $1,244,031$ & 95.96 & 13.63 & $1,033,606$ & 83.09 & 983,376 & 86.38 \\
\hline
\end{tabular}

${ }^{a}$ Conditions are defined based on with or without PCR amplification of DNA library after the first round of capture

bUniqueness and relevant statistics are calculated from reads with MAPQ $\geq 20$ 

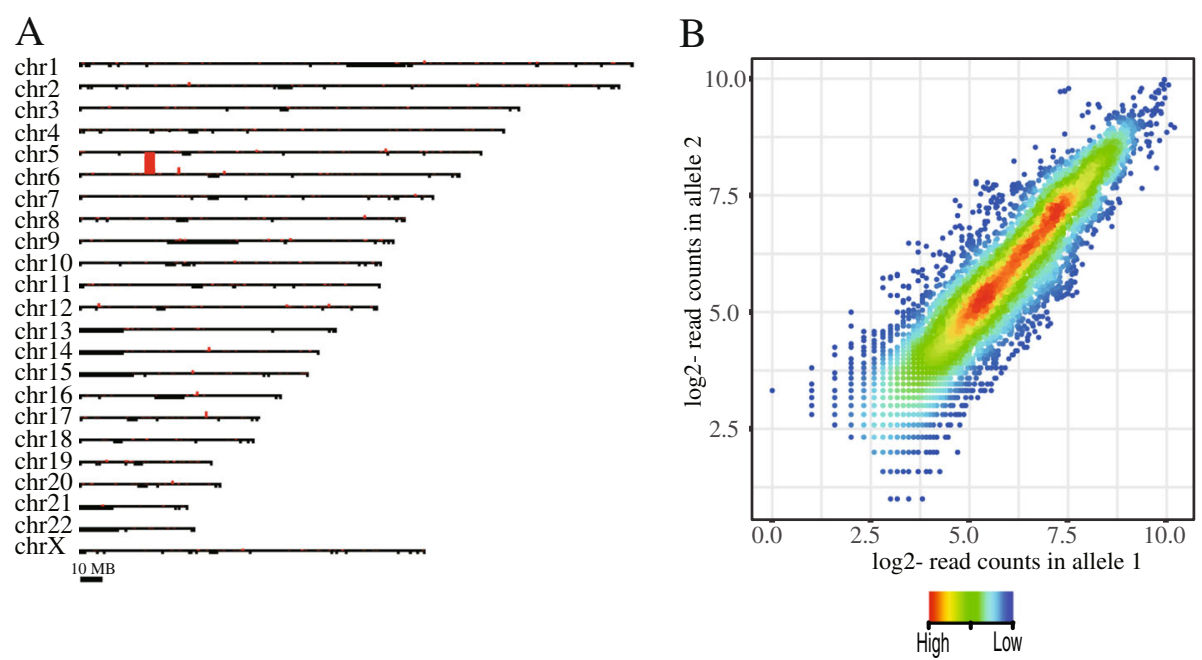

Fig. 3 Double capture and sequencing of the MHC region in the GM12878 genome. a Genome-wide distribution of read coverage in GM12878 genome for dCATCH-Seq. Red bars shown above chromosomes represent the read coverage in $5 \mathrm{~kb}$ windows. Black bars shown below chromosomes represent the gap (unassembled) regions in reference human genome. b Scatterplot of read counts (log2-transformed) for both alleles called among heterozygous variants called within the MHC region. Colors in a rainbow mode represent the density of heterozygous variants

with microarray calls. Overall, the genotyping accuracy was $\sim 99 \%$ in agreement between the dCATCH-Seq approach and microarray data, which is consistent with our previous observations shown above.

We successfully obtained genotypic information for 26 HLA genes in two cell genomes and determined HLA typing with dCATCH-Seq data (Additional file 1: Table S3). To evaluate the accuracy of HLA genotyping, we compared our results with a previous report in the GM12878 genome [20]. The results showed that among 15 typed HLA genes, 13 genes were concordantly typed, especially the five classic HLA class I (HLA-B, C) and HLA class II (HLA-DQA1, DQB1, DRB1) genes that were further validated in this previous study [20]. Taken together, regardless of selected genomic features and size, dCATCHSeq is a robust method to capture any DNA sequences with extremely high target specificity with no substantial diminution of library diversity and introduced bias.

\section{Double capture followed by bisulfite sequencing}

Targeted bisulfite sequencing technology is a robust approach to capture regional DNA methylation levels [21]. To demonstrate the utility of the double capture approach coupled with bisulfite sequencing, we used the dCATCH-Seq procedure on the same targets shown above followed by bisulfite conversion and sequencing. Due to the severe effect of library degradation by bisulfite treatment [22], additional PCR cycles are required to amplify the bisulfite-treated DNA library (e.g., > 20 cycles), which results in high PCR duplication rate in the bisulfite sequencing data. We first determined whether the PCR amplification would introduce bias in quantifying DNA methylation levels. We compared the DNA methylation levels based on a read coverage threshold of at least 10 between two conditions (deduplication of PCR amplified reads or not). The sequencing results for both conditions tested in two cells were summarized in Additional file 1: Table S4. A comparative analysis showed a high concordance rate of $\mathrm{CpG}$ methylation levels between the two conditions across both the chr11 region $\left(n=2666, \mathrm{R}^{2}=0.99, P<1 \times 10^{-16}\right.$, Additional file 2: Figure S8A) and the MHC region $\left(n=3942, \mathrm{R}^{2}=0.97, P<1 \mathrm{X} 10^{-16}\right.$, Additional file 2: Figure $\mathrm{S} 8 \mathrm{C}$ ). Similar patterns were found when comparing non-CpG methylation calls in either $\mathrm{CHG}$ or $\mathrm{CHH}$ dinucleotide context on both regions (Additional file 2: Figure S8B and Figure S8D).

Lastly, we compared DNA methylation profiles between dCATCH-Seq and whole-genome bisulfite sequencing (WGBS) data in the same cell lines. We used the targeted region on chr11 as an example, and the result showed a high concordance $\left(n=208, \mathrm{R}^{2}=0.82\right.$, $P<1 \mathrm{X} 10^{-16}$, Fig. 4 a) of CpG methylation levels detected by both methods. Similarly, both determined a low DNA methylation profiling of the $\mathrm{CHG}$ and $\mathrm{CHH}$ sites (Fig. 4b and c), which are predominantly found present in brain tissue and embryos [23-25]. A similar pattern with a slightly less correlation $\left(R^{2}=0.65, P<1 \times 10^{-16}\right.$, Fig. $4 d$ and e) was observed across the MHC region in GM12878 cells. Altogether, we conclude that the dCATCH-Seq method could also be used to investigate DNA methylation profiles across any genomic regions of interest, and additional PCR cycles did not introduce bias in methylation calls. 


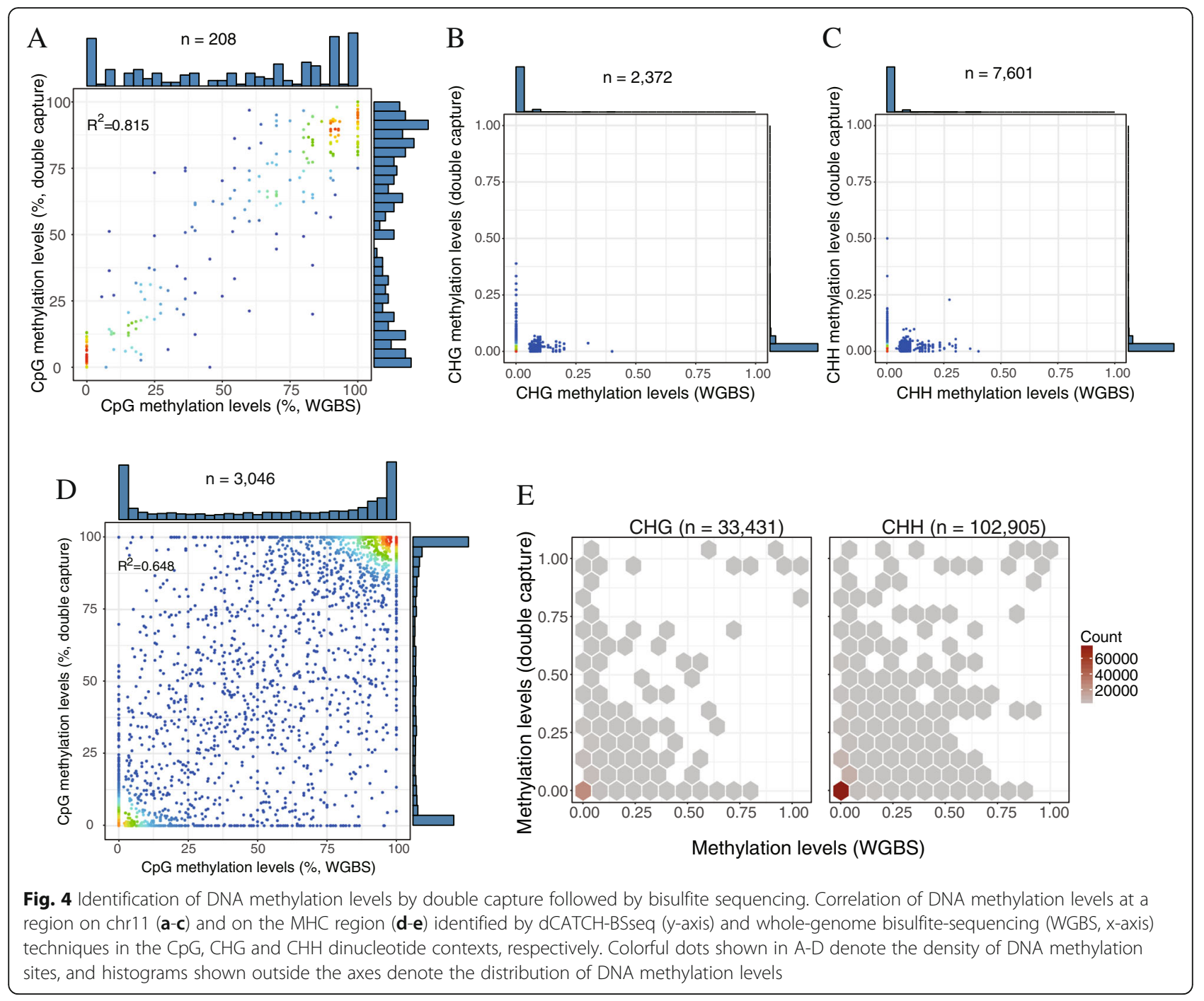

\section{Discussion}

For efficient targeted sequencing for research and diagnostic purposes, capture of DNA or RNA sequence with high specificity is critical. In this study, we presented an improved targeted DNA sequencing approach by using a strategy of two rounds of hybrid selection. Relative to our previous and other reports about the target specificity [9-15], the present approach significantly increases the efficiency of target enrichment. A previous study also used a similar strategy, but captured small, noncontinuous and less complex genomic intervals $(<20 \mathrm{~kb})$ with array-based synthesized probes [26]. We also demonstrate high accuracy and reproducibility of the dCATCH-Seq method in DNA methylation quantitative analyses.

Owing to different design and purpose on capturing genomic regions, a comparison of the dCATCH-Seq method with other target enrichment platforms, e.g. exome enrichment, is generally inappropriate. Given that the enrichment technique for some commercial target enrichment platforms [7], including TruSeq (Illumina), SureSelect (Agilent), SeqCap (Roche NimbleGen), xGen (IDT), MYbaits (MYcroarray) and FleXelect (FlexGen), is similar with the dCATCH-Seq, all based on the hybrid capture, we speculate that the strategy of two-round hybridization approach might be plausible for these hybridization-based target enrichment platforms regardless of target size.

Capture of the entire MHC region by dCATCH-Seq with high efficiency is one of the most promising applications in this study. Because this region is broadly associated with numerous diseases, including cancers [27-29], autoimmune [30-32] and infectious diseases [33], etc. Diverse genetic interactions among MHC, Killer-cell Immunoglobulin-like Receptors (KIRs) and T-cell Receptors (TCRs) are also reported in human populations [34-36].

Several additional efforts could be taken to extend the application of our method with other techniques, such 
as targeted RNA-seq [37], single cell targeted sequencing [38], hybrid capture of viral or transgene integration sites in host genomes [39], enrichment of the human ancient DNA extracted from archaeological samples (e.g., bones) [40], cross-species DNA capture of ultraconserved elements (UCEs) or mitochondrial genomes for phylogenomic studies [41-43]. Conversely, the double capture approach is probably also useful for negative selection to remove unwanted molecules or by-products, such as contaminating DNA within a sample. For example, it is possible to apply the double capture approach to maximally eliminate the rRNA molecules for total RNA-seq [44]. Likewise, because there are approximately $30-50 \%$ of sequenced reads mapped to the mitochondrial genome for transposase-accessible chromatin with sequencing (ATAC-seq) technology [45, 46], the double capture strategy could be probably applicable to remove the mitochondrial DNA sequence.

Finally, some limitations remain in this study. The first limitation is the unequal coverage depth across targeted regions, which is a common issue for the target enrichment methods [7]. Two potential factors could explain this issue. One factor might be due to the usage of blocking reagents, a common and necessary strategy to reduce the background signal for hybridization-based assays. The other factor could be due to the inherent DNA sequences, including $G+C$ content, number and types of repetitive elements across targeted regions. The second limitation is the applicability and efficiency of the double-capture strategy for low-input DNA samples, particularly when the input DNAs down to the levels of tens of nanogram. Because the low amount of DNA input will substantially increase the PCR duplicates in sequencing data and subsequently reduce the superiority for the double-capture approach. Such trade-off between PCR duplicate rates and DNA amount has also been reported in previous target enrichment method [47].

\section{Conclusions}

In conclusion, the $\mathrm{dCATCH}-\mathrm{Seq}$ approach is a powerful and scalable approach to interrogate genetic and epigenetic features on genomic regions of interest, and has the potential to be further combined with other functional genomics approaches.

\section{Methods}

\section{Cell culture and DNA extraction}

GM12878 B-lymphoblast, K562 (a generous gift from Dr. Myer's lab at HudsonAlpha Institute) and U937 cell lines (purchased from ATCC, cat. no. CRL-1593.2) were cultured in RPMI-1640 medium, supplemented with $10 \%$ fetal bovine serum (FBS), $2 \mathrm{mM} \mathrm{L}$-glutamine and $1 \%$ penicillin-streptomycin in a $37{ }^{\circ} \mathrm{C}$ incubator with $5 \%$ $\mathrm{CO}_{2}$. Cells were collected when grown to $\sim 90 \%$ confluence. Following three PBS washes, genomic DNA was extracted using proteinase $\mathrm{K}$ and phenol-chloroform method. Genomic DNA concentration was determined by Qubit fluorometer (ThermoFisher).

\section{Enrichment of targets by two rounds of hybridization}

All BAC-based probes across intended regions were obtained from Ubiquity Genomics, Inc. DNA library construction, target capture and enrichment were previously described [13] with a few modifications. Briefly, input DNA quantities of $1 \mu \mathrm{g}$ and $3 \mu \mathrm{g}$ were used for standard and bisulfite converted dCATCH-Seq approaches, respectively. DNAs were sheared to $\sim 250 \mathrm{bp}$ in size using the Covaris-S220, followed by end-repair, dA-tailing and paired-end Illumina adapter (methylated adapters used for bisulfite sequencing) ligation using NEBNext reagents or Biodynami NGS DNA library prep kit. For the first capture, a hybridization reaction was assembled containing $1 \times$ library DNA, 30× human Cot- 1 DNA (American Genetics), 2500× BAC-derived DNA probes, and $1 \times$ hybridization buffer and denatured at $95{ }^{\circ} \mathrm{C}$ for 5 min followed by incubation of the reaction at $65{ }^{\circ} \mathrm{C}$ for $\sim 48 \mathrm{~h}$. The first capture was performed according to our previous protocol with streptavidin-coated Dynabeads (ThermoFisher), that were washed once at room temperature for $10 \mathrm{~min}$ in $1 \times$ SSC with $0.1 \%$ SDS and twice at $65{ }^{\circ} \mathrm{C}$ for $10 \mathrm{~min}$ in $0.1 \times$ SSC with $0.1 \%$ SDS. After the first capture, an optional PCR enrichment step of the captured library was included for the dCATCHSeq method, with 2X KAPA HiFi master mix (KAPA Biosystems) under the PCR conditions: $45 \mathrm{~s}$ at $98^{\circ} \mathrm{C}$; ten cycles of $15 \mathrm{~s}$ at $98{ }^{\circ} \mathrm{C}, 30 \mathrm{~s}$ at $60^{\circ} \mathrm{C}$, and $45 \mathrm{~s}$ at $72{ }^{\circ} \mathrm{C}$. The hybridization conditions for the second capture were identical with the first, except for incubation of the hybridization reaction at $65{ }^{\circ} \mathrm{C}$ for $\sim 24 \mathrm{~h}$, and the captured samples were washed twice at room temperature by $10 \mathrm{~min}$ in $1 \times \mathrm{SSC}$ with $0.1 \%$ SDS and three times at $65{ }^{\circ} \mathrm{C}$ by $10 \mathrm{~min}$ in $0.1 \times$ SSC with $0.1 \%$ SDS. Finally, DNA was amplified by PCR using indexed primer pairs using the same PCR conditions as above. For the dCATCH-Seq bisulfite sequencing approach, the captured DNA library was bisulfite converted using the EpiTect Bisulfite kit (Qiagen) according to the manufacturer's instructions for small quantities of fragmented DNA. The bisulfite-treated DNA was amplified by using 5 U Platinum Taq DNA Polymerase (ThermoFisher) under the PCR conditions: $98{ }^{\circ} \mathrm{C}$ for $1 \mathrm{~min}$, followed by $20-26$ cycles of $\left(95{ }^{\circ} \mathrm{C}\right.$ for $30 \mathrm{~s}$ and $62{ }^{\circ} \mathrm{C}$ for $3 \mathrm{~min})$. We confirmed the amplification and correct product size range by gel electrophoresis on a $1.7 \%$ agarose gel. Amplified libraries were purified with SPRI beads, and library sizes determined by Agilent Bioanalysis DNA high sensitivity (Agilent), and library concentrations quantified by KAPA Quant kit for Illumina (KAPA 
Biosystems). Libraries were sequenced according to standard Illumina protocol on MiSeq or HiSeq 2500 sequencers.

\section{Public data collection}

We collected (1) microarray genotyping data with two replicates (sample ID: GSM1028244 and GSM1028245) for the U937 cell line, (2) WGBS data for K562 (sample ID: GSE86747) and GM12878 cell lines (sample ID: GSM1002650), and (3) SVs data from a whole-genome sequencing study for K562 cells [18].

\section{Data processing and statistics}

Reads were demuxed based on their index sequence at the Genomic Services Laboratory at HudsonAlpha. After removal of adapter sequences, low-quality reads, and trimmed reads that were shorter than 20 bp by using cutadapt (v1.3.1), filtered reads were aligned with bowtie2 (version 2.1.0) [48] to the human hg19 reference genome. Reads were re-aligned, recalibrated and SNVs and Indels were called using the GATK toolkit (version 3.3) [49]. Variants were filtered for quality as previously described [11]. Briefly, we filtered out variants as follows: (1) mapping quality score $<20$; (2) $\geq 3$ SNPs detected within 10 bp distance; (3) variant confidence/quality by depth < 2; (4) strand bias score > 50; (5) genotype score $<15$; (6) read depth $<10$. The reads per million (RPM) was calculated as the read counts per 200-bp non-overlapping window aligned on the target region divided by per million reads scaling factor. Read mapping was visualized in the Integrative Genomics Viewer (IGV) software [50]. With BAM files as input, HLA genes were typed using the SOAP-HLA [15] with default parameters. Structural variants were called by using Pindel [51] with $\geq 4$ supporting reads and $\geq 100 \mathrm{bp}$ in size. The sequencing data from the bisulfite protocol with dCATCH-Seq and WGBS were aligned and DNA methylation levels at $\mathrm{CpGs}$ and non-CpGs (CHG and $\mathrm{CHH}$ dinucleotides) were estimated by using bismark (version v0.14.1) with default parameters [52]. CpGs, CHGs or CHHs with at least 10X coverage were retained for comparative analyses. Except for relevant programs described, all other bioinformatics analyses were implemented using customized Perl scripts and R programming. All customized codes are available upon request.

\section{Verification of structural variation}

Primer pairs were designed (Additional file 1: Table S5 in and Additional file 2: Figure S5) for each SV by using 200-bp sequences flanking both sides of the deletion region. PCR products were amplified with extracted genomic DNA templates from cells with 5 U NEB Taq DNA Polymerase under the PCR conditions: $95{ }^{\circ} \mathrm{C}$ for $3 \mathrm{~min}$, followed by 30 cycles of $95{ }^{\circ} \mathrm{C}$ for $30 \mathrm{~s}, 55{ }^{\circ} \mathrm{C}$ for $30 \mathrm{~s}$ and $72{ }^{\circ} \mathrm{C}$ for $45 \mathrm{~s}$. PCR products were run on $2 \%$ agarose gels.

\section{Additional files}

\begin{abstract}
Additional file 1: Table S1. Summary of targeted sequencing data in multiple replicates for dCATCH-Seq and CATCH-Seq. Table S2. Comparison of Indel calls between dCATCH-Seq and CATCH-Seq. Table S3. HLA gene typing for dCATCH-Seq. Table S4. Summary of bisulfite dCATCH-Seq data compared between two conditions. Table S5: List of PCR primers for verifying SVs. (XLSX 19 kb)
\end{abstract}

Additional file 2: Figure S1 and S2. Visualization of reads mapping to three SNVs (S1) and two Indels (S2) called for dCATCH-Seq (left panel) and $\mathrm{CATCH}$-Seq (right panel) methods. Red-colored arrows show the location of the discordantly called SNVs (S1) and Indels (S2). Figure S3. Visualization of reads mapping to two Indels (A and B) from an independent $\mathrm{CATCH}$-Seq assay in two replicates. Red-colored arrows, the location of the confirmed Indels. Figure S4. SVs in K562 cells. The upper panels are SVs detected by conditions (with or without PCR) after the first capture. The lower panels are SVs identified by a previous study. Figure S5. Two novel deletions in K562 cells. PCR primer pairs for verifying a homozygous deletion (A) and a heterozygous deletion (C), and the agarose gel electrophoresis results (B and D). P, N and M denote the positive, negative bands and DNA marker, respectively. Due to difficulty in design of the specific primers, there is a second (non-specific) band for the N2 lane. Figure S6. Genome-wide read coverage in U937 cell genome using the dCATCH-Seq. Red bars represent the read depth in $5 \mathrm{~kb}$ windows. Black bars mean the gap (unassembled) regions in the reference human genome. Figure $\mathbf{S 7}$. Scatterplot showing read counts (log2-transformed) on two alleles for heterozygous variants across the MHC region in $U 937$ cell genome. Colors in a rainbow mode represent the density of heterozygous variants. Figure $\mathbf{S 8}$. Comparison of DNA methylation levels at a region on chr11 (A-B) and on the $\mathrm{MHC}$ region (C-D) calculated by read counts with (x-axis) or without (y-axis) removal of PCR duplicates in a CpG (left panel) or non-CpG (right panel) dinucleotide context. Histograms ( $A$ and $C$ ) represent the distribution of $C p G$ methylation levels corresponding to $x$ - and $y$-axes, respectively. (ZIP $8432 \mathrm{~kb}$ )

\section{Abbreviations}

dCATCH-Seq: Double CATCH-Seq; KIR: Killer-cell Immunoglobulin-like Receptor; MHC: Major histocompatibility complex; SNP: Single nucleotide polymorphism; SNV: Single nucleotide variant; SV: Structural variant; TCR: T-cell Receptor; RPM: reads per million; WGBS: Whole-genome bisulfite sequencing

\section{Acknowledgments}

We thank the members of the Genomic Services Laboratory at HudsonAlpha for providing sequence data.

\section{Funding}

This study was supported by the HudsonAlpha Institute Fund. The funders had no role in study design, data collection and analysis, decision to publish, or preparation of the manuscript.

\section{Availability of data and materials}

All data generated during the current study are available in the NCBI Sequence Read Archive under Study Accession number SRP114449. All the supporting data are included as Additional Files.

\section{Authors' contributions}

$Y Z$ and JS designed and optimized the method with intellectual contribution from KD and DA. YZ performed the experiments and analyzed the data. JS and $K D$ assisted in the experiments. $Y Z$ prepared the manuscript with revision from JS, KD and DA. All authors read, interpret and approved the final version of the manuscript.

Ethics approval and consent to participate Not applicable. 


\section{Consent for publication}

Not applicable.

\section{Competing interests}

CATCH-Seq is used by both academic and commercial enterprises, including Ubiquity Genomics, which was founded by D. Absher. The remaining authors declare no competing interests.

\section{Publisher's Note}

Springer Nature remains neutral with regard to jurisdictional claims in published maps and institutional affiliations.

Received: 3 April 2017 Accepted: 5 October 2017

Published online: 23 October 2017

\section{References}

1. Bamshad MJ, Ng SB, Bigham AW, Tabor HK, Emond MJ, Nickerson DA Shendure J. Exome sequencing as a tool for Mendelian disease gene discovery. Nat Rev Genet. 2011;12(11):745-55.

2. Fu W, O'Connor TD, Jun G, Kang HM, Abecasis G, Leal SM, Gabriel S, Rieder MJ, Altshuler D, Shendure J, et al. Analysis of 6,515 exomes reveals the recent origin of most human protein-coding variants. Nature. 2013; 493(7431):216-20.

3. Tennessen JA, Bigham AW, O'Connor TD, Fu W, Kenny EE, Gravel S, McGee S, Do R, Liu X, Jun G, et al. Evolution and functional impact of rare coding variation from deep sequencing of human exomes. Science. 2012;337(6090):64-9.

4. Choi M, Scholl Ul, Ji W, Liu T, Tikhonova IR, Zumbo P, Nayir A, Bakkaloglu A, Ozen S, Sanjad S, et al. Genetic diagnosis by whole exome capture and massively parallel DNA sequencing. Proc Natl Acad Sci U S A. 2009;106(45): 19096-101.

5. Vallender EJ. Expanding whole exome resequencing into non-human primates. Genome Biol. 2011;12(9):R87

6. McCormack JE, Harvey MG, Faircloth BC, Crawford NG, Glenn TC, Brumfield RT. A phylogeny of birds based on over 1,500 loci collected by target enrichment and high-throughput sequencing. PLoS One. 2013;8(1):e54848.

7. Mertes F, Elsharawy A, Sauer S, van Helvoort JM, van der Zaag PJ, Franke A Nilsson M, Lehrach $\mathrm{H}$, Brookes AJ. Targeted enrichment of genomic DNA regions for next-generation sequencing. Brief Funct Genomics. 2011;10(6): 374-86.

8. Meienberg J, Zerjavic K, Keller I, Okoniewski M, Patrignani A, Ludin K, Xu Z, Steinmann B, Carrel T, Rothlisberger B, et al. New insights into the performance of human whole-exome capture platforms. Nucleic Acids Res. 2015:43(11):e76

9. Clark MJ, Chen R, Lam HY, Karczewski KJ, Chen R, Euskirchen G, Butte AJ, Snyder M. Performance comparison of exome DNA sequencing technologies. Nat Biotechnol. 2011;29(10):908-14.

10. Asan, Xu Y, Jiang H, Tyler-Smith C, Xue Y, Jiang T, Wang J, Wu M, Liu X, Tian $G$, et al. Comprehensive comparison of three commercial human wholeexome capture platforms. Genome Biol. 2011;12(9):R95.

11. Zhang Y, Li B, Li C, Cai Q, Zheng W, Long J. Improved variant calling accuracy by merging replicates in whole-exome sequencing studies. Biomed Res Int. 2014;2014:319534.

12. Yi X, Liang $Y$, Huerta-Sanchez $E$, Jin $X$, Cuo ZX, Pool JE, Xu X, Jiang H, Vinckenbosch $N$, Korneliussen TS, et al. Sequencing of 50 human exomes reveals adaptation to high altitude. Science. 2010:329(5987):75-8.

13. Day K, Song J, Absher D. Targeted sequencing of large genomic regions with CATCH-Seq. PLoS One. 2014;9(10):e111756.

14. Yigit E, Zhang Q, Xi L, Grilley D, Widom J, Wang J-P, Rao A, Pipkin ME. Highresolution nucleosome mapping of targeted regions using BAC-based enrichment. Nucleic Acids Res. 2013;41(7):e87.

15. Cao $H$, Wu J, Wang $Y$, Jiang $H$, Zhang $T$, Liu $X, X u Y$, Liang $D$, Gao $P$, Sun $Y$, et al. An integrated tool to study MHC region: accurate SNV detection and $\mathrm{HLA}$ genes typing in human $\mathrm{MHC}$ region using targeted high-throughput sequencing. PLoS One. 2013;8(7):e69388.

16. Samuels DC, Han L, Li J, Quanghu S, Clark TA, Shyr Y, Guo Y. Finding the lost treasures in exome sequencing data. Trends Genet. 2013;29(10):593-9.

17. Klonowska K, Handschuh L, Swiercz A, Figlerowicz M, Kozlowski P. MTTE: an innovative strategy for the evaluation of targeted/exome enrichment efficiency. Oncotarget. 2016;7(41):67266-76.

18. Yao F, Ariyaratne PN, Hillmer AM, Lee WH, Li G, Teo ASM, Woo XY, Zhang Z, Chen JP, Poh WT, et al. Long span DNA paired-end-tag (DNA-PET) sequencing strategy for the interrogation of genomic structural mutations and fusionpoint-guided reconstruction of amplicons. PLoS One. 2012;7(9):e46152.

19. Gnirke A, Melnikov A, Maguire J, Rogov P, LeProust EM, Brockman W, Fennell T, Giannoukos G, Fisher S, Russ C, et al. Solution hybrid selection with ultra-long oligonucleotides for massively parallel targeted sequencing. Nat Biotechnol. 2009;27(2):182-9.

20. Dilthey AT, Gourraud P-A, Mentzer AJ, Cereb N, lqbal Z, McVean G. Highaccuracy HLA type inference from whole-genome sequencing data using population reference graphs. PLoS Comput Biol. 2016;12(10):e1005151.

21. Deng J, Shoemaker R, Xie B, Gore A, LeProust EM, Antosiewicz-Bourget J, Egli D, Maherali N, Park IH, Yu J, et al. Targeted bisulfite sequencing reveals changes in DNA methylation associated with nuclear reprogramming. Nat Biotechnol. 2009:27(4):353-60.

22. Miura F, Enomoto Y, Dairiki R, Ito T. Amplification-free whole-genome bisulfite sequencing by post-bisulfite adaptor tagging. Nucleic Acids Res. 2012;40(17):e136.

23. Ramsahoye BH, Biniszkiewicz D, Lyko F, Clark V, Bird AP, Jaenisch R. NonCpG methylation is prevalent in embryonic stem cells and may be mediated by DNA methyltransferase 3a. Proc Natl Acad Sci U S A. 2000; 97(10):5237-42.

24. Lister R, Pelizzola M, Dowen RH, Hawkins RD, Hon G, Tonti-Filippini J, Nery JR, Lee L, Ye Z, Ngo QM, et al. Human DNA methylomes at base resolution show widespread epigenomic differences. Nature. 2009;462(7271):315-22.

25. Guo JU, Su Y, Shin JH, Shin J, Li H, Xie B, Zhong C, Hu S, Le T, Fan G, et al. Distribution, recognition and regulation of non-CpG methylation in the adult mammalian brain. Nat Neurosci. 2014;17(2):215-22.

26. Schmitt MW, Fox EJ, Prindle MJ, Reid-Bayliss KS, True LD, Radich JP, Loeb LA Sequencing small genomic targets with high efficiency and extreme accuracy. Nat Meth. 2015;12(5):423-5.

27. Shukla SA, Rooney MS, Rajasagi M, Tiao G, Dixon PM, Lawrence MS, Stevens J, Lane WJ, Dellagatta JL, Steelman S, et al. Comprehensive analysis of cancer-associated somatic mutations in class I HLA genes. Nat Biotechnol. 2015;33(11):1152-8.

28. Gragert L, Fingerson S, Albrecht M, Maiers M, Kalaycio M, Hill BT. Finemapping of HLA associations with chronic lymphocytic leukemia in US populations. Blood. 2014;124(17):2657-65.

29. Beksac M, Gragert L, Fingerson S, Maiers M, Zhang MJ, Albrecht M, Zhong X, Cozen W, Dispenzieri A, Lonial S, et al. HLA polymorphism and risk of multiple myeloma. Leukemia. 2016;30(11):2260-4.

30. Raychaudhuri S, Sandor C, Stahl EA, Freudenberg J, Lee HS, Jia X, Alfredsson $L$, Padyukov $L$, Klareskog L, Worthington J, et al. Five amino acids in three HLA proteins explain most of the association between MHC and seropositive rheumatoid arthritis. Nat Genet. 2012;44(3):291-6.

31. Lenz TL, Deutsch AJ, Han B, Hu X, Okada Y, Eyre S, Knapp M, Zhernakova A, Huizinga TW, Abecasis $G$, et al. Widespread non-additive and interaction effects within HLA loci modulate the risk of autoimmune diseases. Nat Genet. 2015:47(9):1085-90.

32. Zhou F, Cao H, Zuo X, Zhang T, Zhang X, Liu X, Xu R, Chen G, Zhang Y, Zheng $X$, et al. Deep sequencing of the $M H C$ region in the Chinese population contributes to studies of complex disease. Nat Genet. 2016;48(7): 740-6.

33. Trowsdale J, Knight JC. Major histocompatibility complex genomics and human disease. Annu Rev Genomics Hum Genet. 2013;14:301-23.

34. Parham P, Moffett A. Variable NK cell receptors and their MHC class I ligands in immunity, reproduction and human evolution. Nat Rev Immunol. 2013; 13(2):133-44.

35. Marrack P, Scott-Browne JP, Dai S, Gapin L, Kappler JW. Evolutionarily conserved amino acids that control TCR-MHC interaction. Annu Rev Immunol. 2008;26:171-203.

36. Norman PJ, Hollenbach JA, Nemat-Gorgani N, Guethlein LA, Hilton HG, Pando MJ, Koram KA, Riley EM, Abi-Rached L, Parham P. Co-evolution of human leukocyte antigen (HLA) class I ligands with killer-cell immunoglobulin-like receptors (KIR) in a genetically diverse population of sub-Saharan Africans. PLoS Genet. 2013:9(10):e1003938.

37. Mercer TR, Gerhardt DJ, Dinger ME, Crawford J, Trapnell C, Jeddeloh JA, Mattick JS, Rinn JL. Targeted RNA sequencing reveals the deep complexity of the human transcriptome. Nat Biotechnol. 2011;30(1):99-104.

38. Leung ML, Wang Y, Waters J, Navin NE. SNES: single nucleus exome sequencing. Genome Biol. 2015;16:55.

39. Duncavage EJ, Magrini V Becker N Armstrong JR, Demeter RT, Wylie T, Abel $H J$, Pfeifer JD. Hybrid capture and next-generation sequencing identify viral 
integration sites from formalin-fixed, paraffin-embedded tissue. J Mol Diagn. 2011;13(3):325-33.

40. Reich D, Green RE, Kircher M, Krause J, Patterson N, Durand EY, Viola B, Briggs AW, Stenzel U, Johnson PL, et al. Genetic history of an archaic hominin group from Denisova cave in Siberia. Nature. 2010;468(7327):1053-60.

41. Faircloth BC, Branstetter MG, White ND, Brady SG. Target enrichment of ultraconserved elements from arthropods provides a genomic perspective on relationships among hymenoptera. Mol Ecol Resour. 2015;15(3):489-501.

42. Jones MR, Good JM. Targeted capture in evolutionary and ecological genomics. Mol Ecol. 2016;25(1):185-202.

43. Mason VC, Li G, Helgen KM, Murphy WJ. Efficient cross-species capture hybridization and next-generation sequencing of mitochondrial genomes from noninvasively sampled museum specimens. Genome Res. 2011;21(10): 1695-704.

44. Ameur A, Zaghlool A, Halvardson J, Wetterbom A, Gyllensten U, Cavelier L, Feuk L. Total RNA sequencing reveals nascent transcription and widespread co-transcriptional splicing in the human brain. Nat Struct Mol Biol. 2011; 18(12):1435-40,

45. Sos BC, Fung H-L, Gao DR, Osothprarop TF, Kia A, He MM, Zhang K. Characterization of chromatin accessibility with a transposome hypersensitive sites sequencing (THS-seq) assay. Genome Biol. 2016;17(1):20.

46. Tsompana M, Buck MJ. Chromatin accessibility: a window into the genome. Epigenetics Chromatin. 2014;7(1):33.

47. Rohland N, Reich D. Cost-effective, high-throughput DNA sequencing libraries for multiplexed target capture. Genome Res. 2012;22(5):939-46.

48. Langmead B, Salzberg SL. Fast gapped-read alignment with bowtie 2. Nat Meth. 2012;9(4):357-9.

49. DePristo MA, Banks E, Poplin R, Garimella KV, Maguire JR, Hartl C, Philippakis AA, del Angel G, Rivas MA, Hanna M, et al. A framework for variation discovery and genotyping using next-generation DNA sequencing data. Nat Genet. 2011;43(5):491-8.

50. Robinson JT, Thorvaldsdottir H, Winckler W, Guttman M, Lander ES, Getz G, Mesirov JP. Integrative genomics viewer. Nat Biotechnol. 2011;29(1):24-6.

51. Ye K, Schulz MH, Long Q, Apweiler R, Ning Z. Pindel: a pattern growth approach to detect break points of large deletions and medium sized insertions from paired-end short reads. Bioinformatics. 2009;25(21):2865-71.

52. Krueger F, Kreck B, Franke A, Andrews SR. DNA methylome analysis using short bisulfite sequencing data. Nat Meth. 2012;9(2):145-51.

\section{Submit your next manuscript to BioMed Central and we will help you at every step:}

- We accept pre-submission inquiries

- Our selector tool helps you to find the most relevant journal

- We provide round the clock customer support

- Convenient online submission

- Thorough peer review

- Inclusion in PubMed and all major indexing services

- Maximum visibility for your research

Submit your manuscript at www.biomedcentral.com/submit

C) Biomed Central 